\title{
Moment of inertia, quadrupole moment, Love number of neutron star and their relations with strange matter equations of state
}

\author{
Debades Bandyopadhyay ${ }^{1}$, Sajad A. Bhat ${ }^{1}$, Prasanta Char ${ }^{2}$ and Debarati Chatterjee ${ }^{3}$ \\ 1 Saha Institute of Nuclear Physics, HBNI, 1/AF Bidhannagar, Kolkata-700064, India \\ 2 Inter-University Centre for Astronomy and Astrophysics, Post Bag 4, Ganeshkhind, Pune - 411007 \\ 3 LPC/ENSICAEN, 6 Boulevard Marechal Juin, 14050, Caen, France \\ Received: date / Revised version: date
}

\begin{abstract}
We investigate the impact of strange matter equations of state involving $\Lambda$ hyperons, BoseEinstein condensate of $K^{-}$mesons and first order hadron-quark phase transition on moment of inertia, quadrupole moment and tidal deformability parameter of slowly rotating neutron stars. All these equations of state are compatible with the $2 \mathrm{M}_{\text {solar }}$ constraint. The main findings of this investigation are the universality of the I-Q and I-Love number relations, which are preserved by the EoSs including $\Lambda$ hyperons and antikaon condensates, but broken in presence of a first order hadron-quark phase transition. Furthermore, it is also noted that the quadrupole moment approaches the Kerr value of a black hole for maximum mass neutron stars.
\end{abstract}

PACS. 9 7.60.Jd neutron stars - 26.60.Kp equations of state

\section{Introduction}

Dense matter equation of state (EoS) is a key ingredient to understanding physics of core-collapse supernovae, neutron stars and merger of neutron stars. The determination of EoS of dense matter is a challenging task for the scientific community. Low density matter below the saturation density might be constrained using inputs from nuclear physics experiments in laboratories. On the other hand, the EoS at several times normal nuclear matter density is probed by astrophysical observations.

Neutron stars are investigated in multi-wavelength observations. These resulted in macroscopic properties of neutron stars, for example, masses, surface magnetic fields and temperatures. Mass measurement in neutron star binaries particularly relativistic binaries involving pulsars has reached a high precision level. The highest neutron star mass measured most accurately so far is $2.01 \pm 0.04$ $\mathrm{M}_{\text {solar }}$ 1]. This puts severe constraint on the EoS of dense matter. Radius measurement is still a debatable issue. Efforts to extract information about radius due to surface emission in x-ray thermonuclear bursts and from accreting neutron stars in quiescence are going on, but complicated by uncertainties in the composition of atmosphere and distance to sources. Recently launched Neutron Star Composition Explorer (NICER) Mission could provide a better estimation of neutron radius observing and analysing soft $\mathrm{x}$-rays from rotation powered pulsars if the mass is known accurately. An alternative to this is the measurement of

Correspondence to: debades.bandyopadhyay@saha.ac.in moment of inertia (I), for example that of pulsar A in double pulsar system PSR J0737-3039 2,3. There will be many fold increase in the number of newly discovered pulsars in relativistic binaries with the advent of highly sensitive Square Kilometre Array (SKA). This will facilitate faster estimation of moment of inertia in the SKA era. Consequently, simultaneous knowledge of mass and radius of same neutron star will be available. Furthermore, this might lead to the better understanding of EoS in neutron star interior.

After the discoveries of black hole and neutron star merger events, gravitational waves open up a new vista into astrophysical observations. It is expected that gravitational wave detectors such as Advanced LIGO, VIRGO or LIGO-India 4] might record gravitational waves from neutron star merger events. Gravitational waves from merging neutron stars provide an interesting opportunity to probe dense matter in neutron star interior and its EoS [5, 6. In the late inspiralling phase of binary neutron stars, tidal deformations could be estimated. This tidal deformation depends on the EoS. The tidal deformation is described by a set of parameters known as Love numbers. The quadrupole Love number $\left(k_{2}\right)$ is an important quantity which is given by the ratio of quadrupole and tidal tensors. It might be possible to extract the Love number from the detection of gravitational wave signal $[7,8$, 9 . The recent investigation on this issue found that the Love number was related to moment of inertia and spin induced quadrupole moment $(Q)$ through universal relations [10]. 
The core motivation to find a universal relation was to describe the exterior spacetime of a compact object independently of its internal structure, as the lack of experimental and observational findings results in poor knowledge of the interior of those objects. However, to use these relations one must be careful to incorporate the proper physical conditions where these are valid. For example, it is found that at high rotational frequencies $\sim 1 k \mathrm{~Hz}$ [11 and high magnetic field $\sim 10^{13} G[12$ the universal relations are broken. The usage and implications of the so called I-Love-Q relations can be found in the recent review by Yagi and Yunes [13] and references therein. However, we find the lack of evidences in the literature regarding whether these relations will also hold strong in presence of a first order phase transition inside the neutron star or not. This motivates us to look into this problem.

Observed masses, radii and moments of inertia are direct probes of compositions and EoS of dense matter in neutron stars. The appearance of strange matter in the form of novel phases of hyperons, Bose-Einstein condensate of antikaons and quarks are highly plausible above 2-3 times normal nuclear matter density in neutron star interior. Strange matter whether it is hyperon matter [14, 15, 16, 17, kaon condensed matter [18, 19] or quark matter 20, makes the EoS softer and results in reduction in maximum mass compared with that of neutron stars made of neutrons and protons only. Keeping pace with latest observations, EoS models have to explain two solar mass or more massive neutron stars. Strange matter EoS models encounter a precarious situation. For hyperons, this is known as the hyperon puzzle. This puzzle could be solved by introducing an extra repulsion into the EoS [16].

Motivated by the recent advances in multi-wavelength observations of neutron stars and the detection of gravitational waves in neutron star merger, we investigate dense matter EoSs involving strangeness degrees of freedom and their impact on the moment of inertia, quadrupole moment and love number. Furthermore, we want to explore whether universal relations among those observables hold good even in presence of exotic matter involving first order phase transition or not.

We use geometrized units $c=G=1$ in this paper. The paper is organised in the following way. We describe dense matter EoSs with various compositions within the framework of a field theoretical model and the calculation of love number, moment of inertia and quadrupole moment in Section 2. Results are discussed in Section 3. We conclude in Section 4.

\section{Equation of State}

We compute the moment of inertia, quadrupole moment and love number in slowly rotating neutron stars. Each of these quantities is dependent on the EoS. The EoS for charge-neutral and beta-equilibrated matter made of baryons, antikaon condensate and leptons is constructed. We adopt a density dependent relativistic hadron (DDRH) field theory for the description of strongly interacting dense baryonic matter. Baryon-baryon interaction in the DDRH

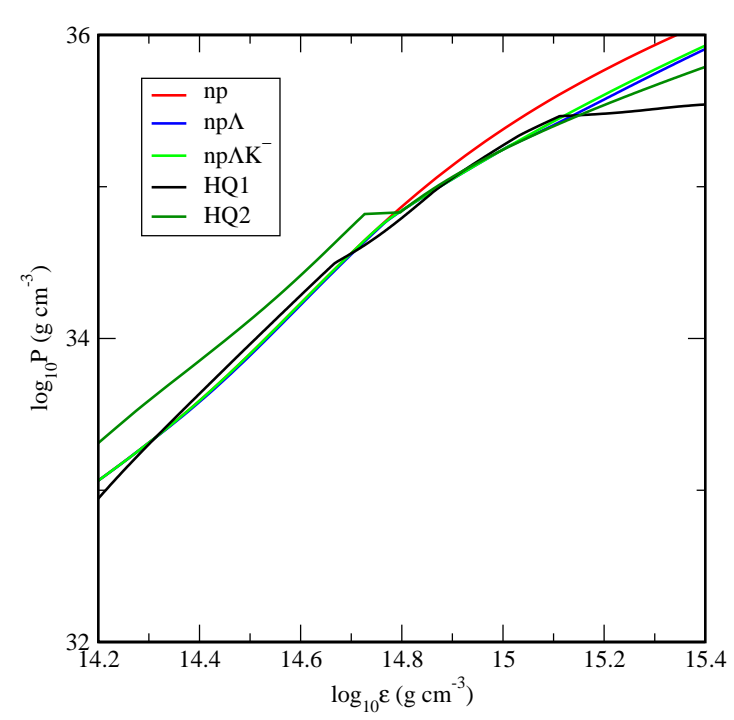

Fig. 1. Pressure versus energy density is plotted for different compositions of matter.

model is mediated by exchanges of scalar $\sigma$, vector $\omega, \phi$ and $\rho$ mesons and is given by the Lagrangian density [21, 22,23 ,

$$
\begin{aligned}
\mathcal{L}_{B}= & \sum_{B} \bar{\psi}_{B}\left(i \gamma_{\mu} \partial^{\mu}-m_{B}+g_{\sigma B} \sigma-g_{\omega B} \gamma_{\mu} \omega^{\mu}-g_{\phi B} \gamma_{\mu} \phi^{\mu}\right. \\
& \left.-g_{\rho B} \gamma_{\mu} \boldsymbol{\tau}_{B} \cdot \boldsymbol{\rho}^{\mu}\right) \psi_{B} \\
& +\frac{1}{2}\left(\partial_{\mu} \sigma \partial^{\mu} \sigma-m_{\sigma}^{2} \sigma^{2}\right)-\frac{1}{4} \omega_{\mu \nu} \omega^{\mu \nu} \\
& +\frac{1}{2} m_{\omega}^{2} \omega_{\mu} \omega^{\mu}-\frac{1}{4} \phi_{\mu \nu} \phi^{\mu \nu}+\frac{1}{2} m_{\phi}^{2} \phi_{\mu} \phi^{\mu} \\
& -\frac{1}{4} \boldsymbol{\rho}_{\mu \nu} \cdot \boldsymbol{\rho}^{\mu \nu}+\frac{1}{2} m_{\rho}^{2} \boldsymbol{\rho}_{\mu} \cdot \boldsymbol{\rho}^{\mu} .
\end{aligned}
$$

Here $\psi_{B}$ stands for the baryon octet, $\boldsymbol{\tau}_{\boldsymbol{B}}$ is the isospin operator and density dependent meson-baryon couplings are denoted by $g \mathrm{~s}$. The exchange of $\phi$ mesons accounts for the repulsive hyperon-hyperon interaction and could be a plausible solution to the hyperon puzzle. Nucleon-nucleon interaction is not mediated by $\phi$ mesons.

Meson field equations are solved in the mean field approximation (MFA). Finally we obtain the pressure versus energy density known as the EoS as given by Ref.[21, 22]. For density dependent couplings, the pressure includes the rearrangement term which takes care of many body effects and thermodynamic consistency.

The effective mass and in-medium energy of antikaons decrease with baryon density. The s-wave Bose-Einstein condensate of antikaons sets in when the electron chemical potential is equal to the in-medium energy of antikaons. 


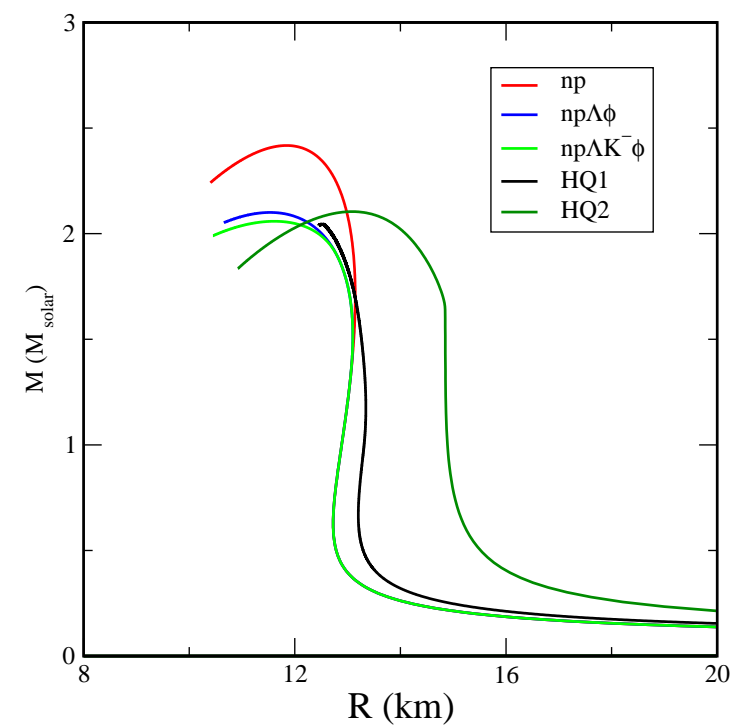

Fig. 2. Mass versus radius is plotted for equations of state shown in Fig. 1.

The phase transition from the hadronic to antikaon condensed phase could be either a first or second order phase transition. In the antikaon condensed phase, baryons are embedded in the condensate. We treat the kaon-baryon interaction in the same footing as the baryon-baryon interaction in Eq. (1). The Lagrangian density for (anti)kaons in the minimal coupling scheme is $22,24,25]$,

$$
\mathcal{L}_{K}=D_{\mu}^{*} \bar{K} D^{\mu} K-m_{K}^{* 2} \bar{K} K,
$$

where $K$ and $\bar{K}$ denote kaon and (anti)kaon doublets; the covariant derivative is $D_{\mu}=\partial_{\mu}+i g_{\omega K} \omega_{\mu}+i g_{\phi K} \phi_{\mu}+$ $i g_{\rho K} \mathrm{t}_{K} \cdot \boldsymbol{\rho}_{\mu}$ and the effective mass of antikaons is $m_{K}^{*}=$ $m_{K}-g_{\sigma K} \sigma$. Solving the equations of motion in the MFA, we obtain the EoS in the antikaon condensed phase. It is to be noted that kaon-meson couplings are not density dependent.

Next we consider two equations of state undergoing first order phase transition from hadronic matter to quark matter [26, 27, 28, In the first case, for the hadronic matter above saturation density, the DDRH model as described above is employed here. This hadronic phase includes all hyperons plus $\Delta$ resonance. A non-local extension of the Nambu-Jona-Lasinio model is adopted to describe the quark phase made of $u, d$ and $s$ quarks [29]. The hadron-quark mixed phase is governed by the Gibbs rules. This hadron-quark (HQ) EoS is denoted by HQ1 hereafter. In the other case, the hadronic phase is composed of only nucleons and described by the NL3 model whereas the effective bag model including quark interaction is adopted for the quark phase 28. The Maxwell construction is implemented for the mixed phase. We call this hadron-quark EoS as HQ2.

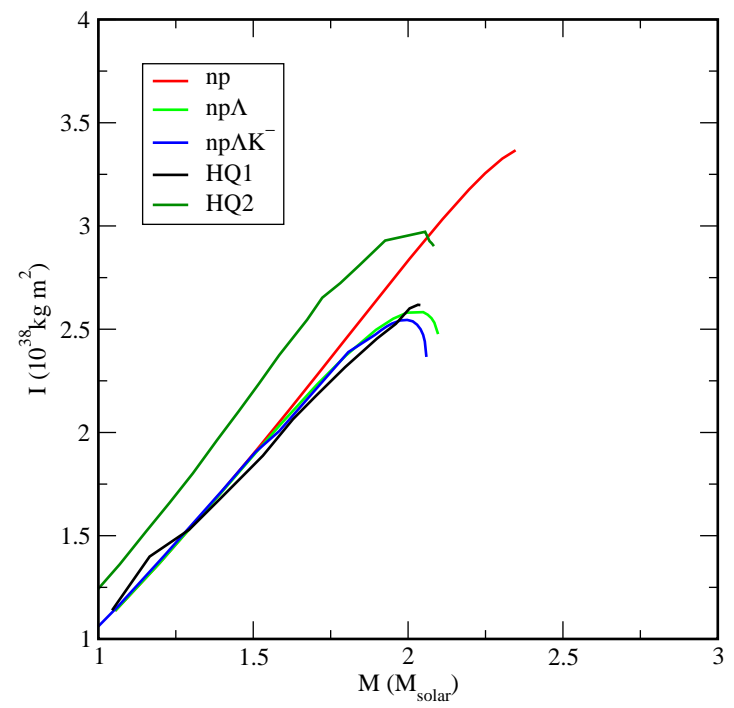

Fig. 3. Moment of inertia is shown as a function of neutron star mass for different compositions of matter.

\subsection{Model of Rotating Neutron Stars}

Most of observed neutron stars rotate slowly. The fastest rotating neutron star has a spin frequency of $716 \mathrm{~Hz}$. Rotation or tidal field makes a neutron star deformed. Here we study rigidly rotating neutron stars assuming a stationary, axisymmetric spacetime and the line element is given by 30,31

$d s^{2}=-N^{2} d t^{2}+A^{2}\left(d r^{2}+r^{2} d \theta^{2}\right)+B^{2} r^{2} \sin ^{2} \theta\left(d \phi-N^{\phi} d t\right)^{2}$,

where metric functions $N, N^{\phi}, A, B$ depend on coordinates $r$ and $\theta$.

The energy-momentum tensor for a perfect fluid which describes the matter, is

$$
T^{\mu \nu}=(\varepsilon+P) u^{\mu} u^{\nu}+P g^{\mu \nu} .
$$

Here the first term represents the matter contribution (perfect fluid), where $\varepsilon$ is the energy density, $P$ is the pressure and $u^{\mu}$ is the fluid four velocity.

The equilibrium configurations for rotating neutron stars are determined by solving the Einstein field equations. For rigid rotation, this is equivalent to solving a first integral of the equation of fluid stationary motion 30 ]

$$
H(r, \theta)+\ln N-\ln \Gamma(r, \theta)=\text { const },
$$

where $\Gamma$ is the Lorentz factor of the fluid with respect to the Eulerian observer and the fluid log-enthalpy is

$$
H=\ln \left(\frac{\varepsilon+P}{n m_{B}}\right) .
$$

Here $n$ and $m_{B}$ are baryon density and rest mass, respectively. 
The first-integral (Eq. 5) is integrable within the $3+1$ formalism, where they reduce to Poisson-like partial differential equations. These equations are then solved numerically using spectral scheme within the numerical library LORENE 32,33. The input parameters for the model are an Equation of State,the rotation frequency $\Omega$ and the central log-enthalpy $H_{c}$. Global properties of rotating neutron stars such as gravitational mass, circumferential equatorial radius, angular momentum, moment of inertia and quadrupole moment are calculated within this formalism using the asymptotic behaviour of lapse function $(N)$ and the component of the shift vector $\left(N^{\phi}\right)$, in terms of metric potential and the source 30,31 . The gravitational mass, angular momentum and quadrupole moment are given respectively as $31,34,35$,

$$
\begin{gathered}
M=\frac{1}{4 \pi} \int \sigma_{\ln N} r^{2} \sin ^{2} \theta d r d \theta d \phi \\
J=\int A^{2} B^{2}(E+p) U r^{3} \sin ^{2} \theta d r d \theta d \phi . \\
Q=-M_{2}-\frac{4}{3}\left(b+\frac{1}{4}\right) M^{3},
\end{gathered}
$$

where,

$$
M_{2}=-\frac{3}{8 \pi} \int \sigma_{\operatorname{lnN}}\left(\cos ^{2} \theta-\frac{1}{3}\right) r^{4} \sin ^{2} \theta d r d \theta d \phi
$$

Here, $\sigma_{\ln N}$ is given by the RHS of Eq. 3.19 of [30, $U$ is the fluid four-velocity, $E=\Gamma^{2}(\varepsilon+p)-p$ and $\Gamma=\left(1-U^{2}\right)^{-1 / 2}$ and $b$ is defined by Eq. (3.37) of 35. Then, the moment of inertia of the rotating star is defined as,

$$
I:=\left|\frac{J}{\Omega}\right|
$$

At lower rotation frequencies, this behaves almost as a linear relation.

\subsection{Love Number, Moment of Inertia and Quadrupole Moment}

When a static, spherically symmetric star is under the influence of a static external quadrupolar tidal field $E_{i j}$, it develops a quadrupole moment $Q_{i j}$. The tidal deformability $\lambda$ in the linear order is defined as

$$
\lambda=-\frac{Q i j}{E_{i j}}
$$

The $\ell=2$ dimensionless tidal Love Number $\left(k_{2}\right)$ is related to the tidal deformability as

$$
k_{2}=\frac{3}{2 R^{5}} \lambda
$$

We calculate the tidal love number following the prescription by Hinderer et al. 8,9. In the Regge-Wheeler

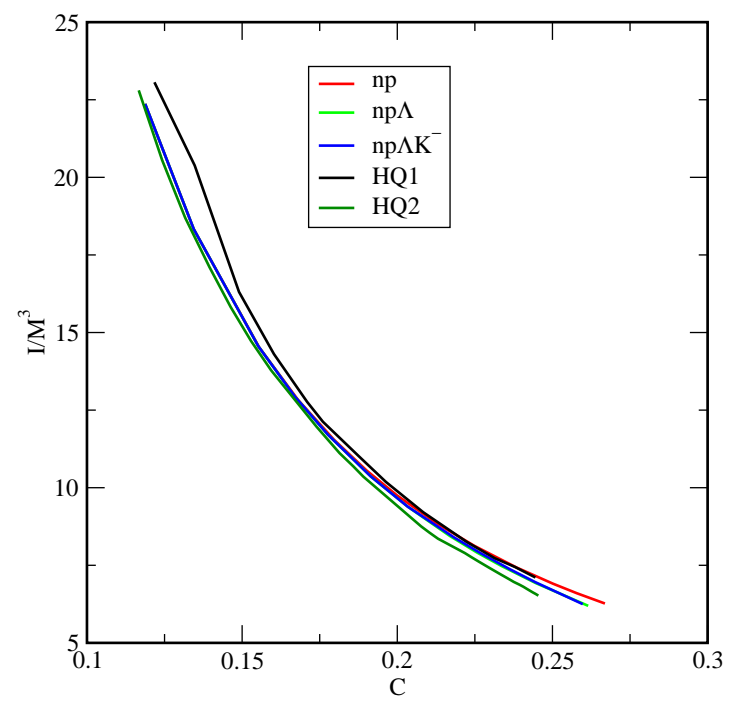

Fig. 4. Dimensionless moment of inertia $\left(\bar{I}=I / M^{3}\right)$ is plotted with compactness.

gauge, a linear, static and even parity $\ell=2, m=0$ perturbation leads to the following form of deformed metric,

$$
\begin{aligned}
d s^{2}= & -e^{2 \Phi(r)}\left[1+H(r) Y_{20}(\theta, \varphi)\right] d t^{2} \\
& +e^{2 \Lambda(r)}\left[1-H(r) Y_{20}(\theta, \varphi)\right] d r^{2} \\
& +r^{2}\left[1-K(r) Y_{20}(\theta, \varphi)\right]\left(d \theta^{2}+\sin ^{2} \theta d \varphi^{2}\right)
\end{aligned}
$$

where, $K^{\prime}(r)=H^{\prime}(r)+2 H(r) \Phi^{\prime}(r)$. This leads to a second order differential equation for metric function $H$

$$
\begin{aligned}
& H^{\prime \prime}+H^{\prime}\left(\frac{2}{r}+\Phi^{\prime}-\Lambda^{\prime}\right)+H\left(-\frac{6 e^{2 \Lambda}}{r^{2}}-2\left(\Phi^{\prime}\right)^{2}\right. \\
& \left.+2 \Phi^{\prime \prime}+\frac{3}{r} \Lambda^{\prime}+\frac{7}{r} \Phi^{\prime}-2 \Phi^{\prime} \Lambda^{\prime}+\frac{f}{r}\left(\Phi^{\prime}+\Lambda^{\prime}\right)\right)=0 .
\end{aligned}
$$

where, $f=d \epsilon / d p$. We solve this equation by integrating outward from the center and using the asymptotic behaviour of $H(r)$, we get the $\ell=2$ tidal love number,

$$
\begin{aligned}
k_{2}= & \frac{8 C^{5}}{5}(1-2 C)^{2}[2+2 C(y-1)-y] \\
& \times\{2 C[6-3 y+3 C(5 y-8)] \\
& +4 C^{3}\left[13-11 y+C(3 y-2)+2 C^{2}(1+y)\right] \\
& \left.+3(1-2 C)^{2}[2-y+2 C(y-1)] \ln (1-2 C)\right\}
\end{aligned}
$$

Here, we define $y=R H^{\prime}(R) / H(R)$ and take compactness of the star, $C=M / R$.

It is to be noted that the tidal deformability parameter, moment of inertia and quadrupole moment are dependent on the EoS individually. However, it was predicted 


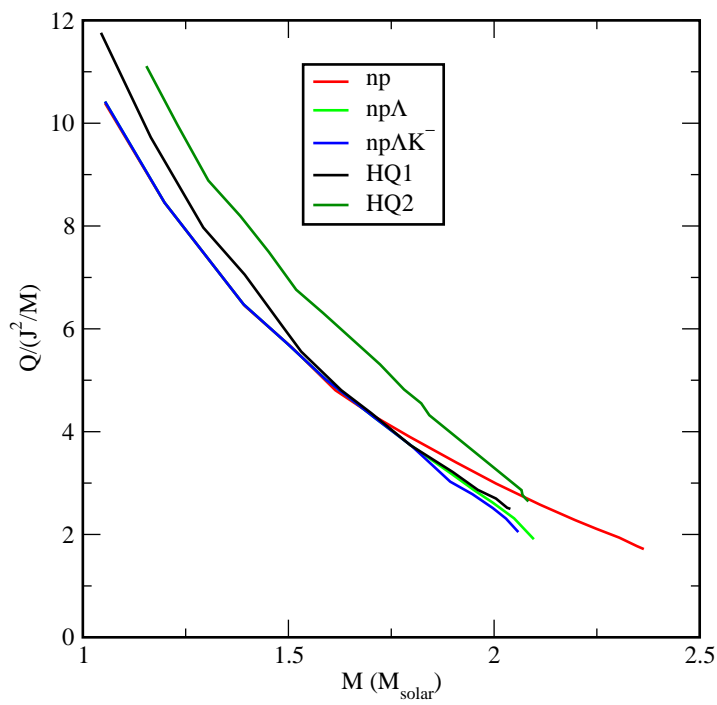

Fig. 5. Dimensionless quadrupole moment with respect to the Kerr solution is plotted with neutron star mass.

that the relation among any two of them in the slow rotation approximation was EoS independent 10. We use the following dimensionless quantities in this investigation. The dimensionless $\lambda$ is defined as

$$
\bar{\lambda}=\frac{\lambda}{M^{5}}
$$

Similarly we introduce dimensionless moment of inertia and quadrupole moment as $\bar{I}=\frac{I}{M^{3}}$ and $\bar{Q}=\frac{Q}{\left(M^{3}\left(J / M^{2}\right)^{2}\right)}$ Here $Q$ is compared with the Kerr solution quadrupole moment $J^{2} / M$. The dimensionless $\mathrm{Q}$ is known as the Kerr factor.

\section{Results and Discussion}

We use the density dependent parameter set referred to as the DD2 set for nucleon-meson couplings 21,23. Hyperonmeson couplings are determined from the SU(6) symmetry relations and hypernuclear data [15,16]. Being lightest among all hyperons, $\Lambda$ hyperons might be populated first in dense matter. We consider only $\Lambda$ hyperons in this calculation. For $\Lambda$ s, we consider a potential depth of $-30 \mathrm{MeV}$ in normal nuclear matter and estimate the scalar meson coupling. Similarly, kaon-meson couplings are determined using the quark model, $\mathrm{SU}(3)$ relations and kaon atomic data 22. In this calculation, an attractive potential depth of $-120 \mathrm{MeV}$ in normal nuclear matter is adopted for the determination of kaon-scalar meson coupling. Parameters of the quark phase are taken from Ref. [26, 28]. With those parameter sets, we calculate various properties of rotating neutron stars as described in section 2 for four different compositions of matter namely neutron-proton matter (denoted as $n p), \Lambda$ hyperon matter $(n p \Lambda)$, antikaon condensed matter including $\Lambda$ hyperons $\left(n p \Lambda K^{-}\right)$and hadron-quark matter.

Figure 1 exhibits the pressure $(P)$ as a function of energy density $(\varepsilon)$ known as the EoS for different compositions of matter. It is evident from the figure that strange matter components such as $\Lambda$ hyperons, antikaons in the condensate or quarks make an EoS softer. Two kinks in both HQ EoSs show the beginning and end of the hadronquark mixed phase [26, 27,28. Among the five EoSs, the HQ1 EoS involving hadron-quark phase transition is the softest EoS and $n p$ matter has the stiffest EoS. We calculate static neutron star structures using those EoSs. The mass-radius relation is shown in Fig. 2, It is found that maximum masses corresponding to $n p, n p \Lambda, n p \Lambda K^{-}$, HQ1 and HQ2 EoS are 2.42, 2.1, 2.06, 2.04 and $2.1 \mathrm{M}_{\text {solar }}$, respectively. It demonstrates that all these EoSs are compatible with the $2 \mathrm{M}_{\text {solar }}$ constraint.

The chance for the measurement of moment of inertia has brightened with the detection of the relativistic pulsar binary PSR J0737-3039. In particular, the estimation of I for pulsar A in the double pulsar system might be possible in near future. Masses of both pulsars in the double pulsar system had been accurately measured due to the knowledge of post Keplerian parameters. The precise measurement of I from observations along with the accurately known mass of pulsar A might overcome the uncertainties in the determination of radius [3]. Here we present our results on the effects of exotic matter on moment of inertia. We are considering slowly rotating neutron stars with spin frequency $100 \mathrm{~Hz}$ in this calculation using LORENE. The behaviour of moment of inertia with neutron star mass is shown in Fig. 3. It is noted that the moment of inertia corresponding to strange matter is lower than that of nuclear matter at higher neutron star masses. As we know the mass (1.337 $\mathrm{M}_{\text {solar }}$ ) of pulsar A in PSR J0737-3039, we can predict the value of I from Fig. 3.

Dimensionless moment of inertia as defined in section 2.2 as a function of compactness is shown in Fig. 4 for four EoSs. It is observed that curves corresponding to $n p, n p \Lambda$ and $n p \Lambda K^{-}$merge before the onset of $\Lambda$ hyperons or antikaon condensate. As soon as strange degrees of freedom appear in dense neutron star matter, $\bar{I}$ for strange matter EoSs deviates from that of the nuclear matter case at higher compactness. However, it is noted that results of $n p \Lambda$ and $n p \Lambda K^{-}$cases show almost a universal behaviour. This may be attributed to the significant population of $\Lambda$ hyperons even after the appearance of antikaon condensate.

We continue our investigation of quadrupole moment of rotating neutron stars having spin frequency of $100 \mathrm{~Hz}$ using LORENE. The behaviour of the quadrupole moment in comparison to the Kerr solution is shown with neutron star mass in Fig. 5. It is found that the quadrupole moment decreases with mass and approaches the Kerr value around maximum neutron star mass. The stiffest EoS corresponding to the nuclear matter case is closest to the Kerr solution. This was already noted by others $36,37,38$. We obtain the most interesting result when we plot dimensionless I versus dimensionless Q in Fig. 6. All model calcu- 


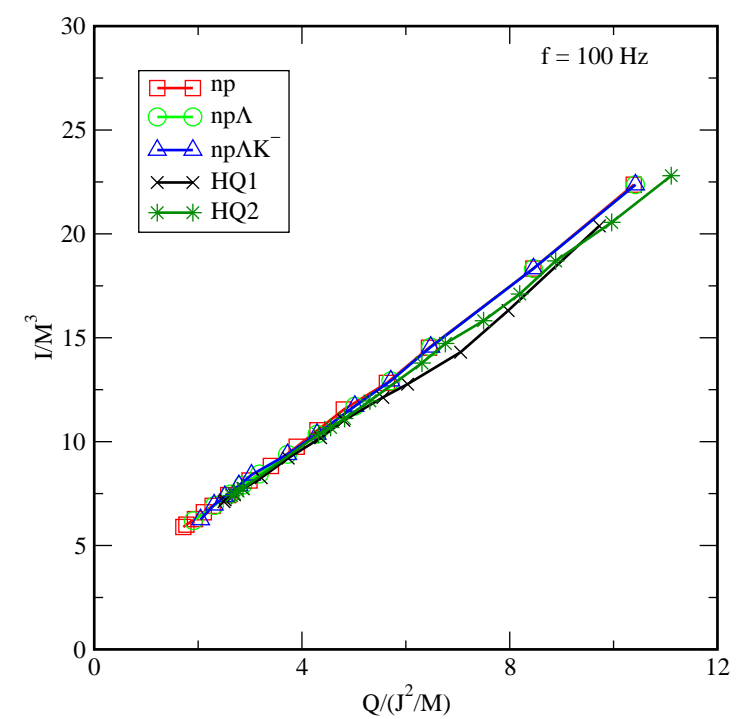

Fig. 6. Dimensionless moment of inertia is shown as a function of dimensionless quadrupole moment for rotational frequency $100 \mathrm{~Hz}$.

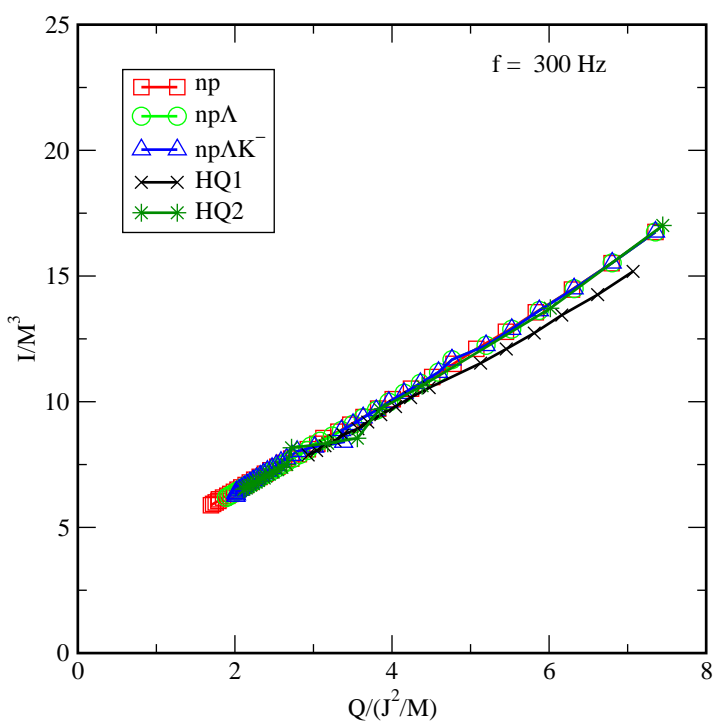

Fig. 7. Same as Fig. 6 but for rotational frequency $300 \mathrm{~Hz}$.

lated data corresponding to different EoSs fall on the same line except that of the HQ EoSs. Though I or Q is sensitive to EoS, their relationship exhibit universality for $n p$, $n p \Lambda$ and $n p \Lambda K^{-}$EoS. This kind of universal relation was first predicted by Yagi and Yunes [10. This holds good for other observable quantities also. When the ratio of critical mass i.e., the maximum mass at the mass-shedding limit and maximum mass for static neutron stars are plotted with normalised angular momentum with respect to maximum angular momentum, it also shows a universal relation 39, 40. However, this universality appears to be lost

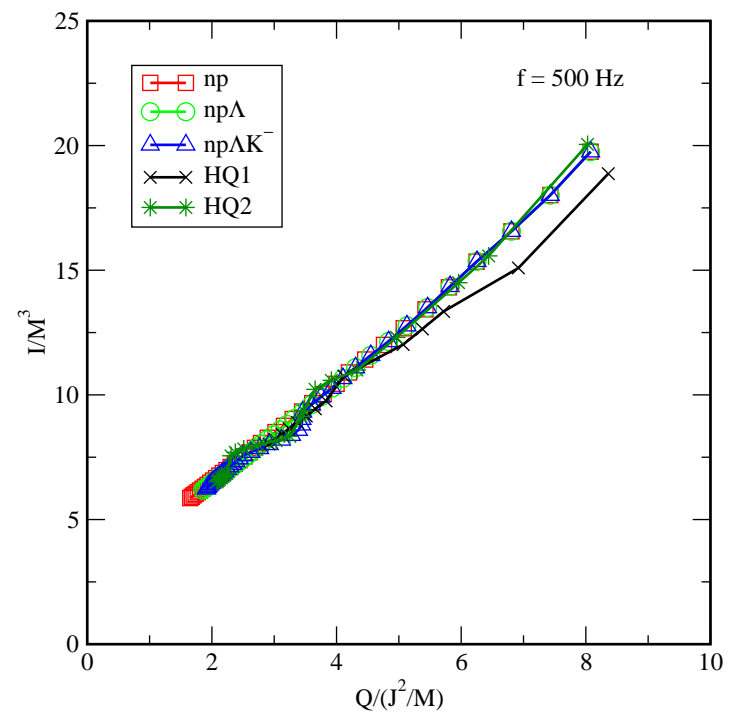

Fig. 8. Same as Fig. 6 but for rotational frequency $500 \mathrm{~Hz}$.

when we look at both HQ EoSs. This may be attributed to a first order hadron-quark phase transition in HQ1 and HQ2.

We further investigate the role of first order phase transition on the I-Q universality relation at higher rotational frequencies. It is a well known fact that the rotation drives compositional changes in neutron stars 26. This has significant implications for the hadron-quark phase transition in rotating neutron stars. As a neutron star spins down, its central density increases from below. When the density exceeds the threshold value, a hadron-quark phase transition sets in. We study I-Q relation for 300 and $500 \mathrm{~Hz}$ to understand the above effect and the results are demonstrated in Fig. 7 and Fig. 8. For HQ2 EoS, the hadronquark phase transition disappears at those frequencies and the universality is retained. However, the imprint of the phase transition can be found even at higher rotational frequencies for HQ1 case. This may be attributed to the fact that HQ1 EoS has a much wider mixed phase compared with that of HQ2 EoS.

Next we study the relation between I and Love number for strange matter EoSs adopting slow rotation approximation of Hartle and Thorne [36. First we plot $\ell=2$ love number $\left(k_{2}\right)$ with compactness $(C=M / R)$ in Fig 9 for $n p, n p \Lambda, n p \Lambda K^{-}$and HQ matter. The love number measures how easy or difficult to deform a neutron star. For each EoS, love number decreases as the compactness increases. This implies that more the star is compact, less is its love number. Furthermore, this effect is more pronounced in softer EoSs i.e. $n p \Lambda, n p \Lambda K^{-}$and HQ than that of $n p$ matter. The love number approaches zero for black holes for which compactness is 0.5 . Figure 10 describes the behaviour of dimensionless moment of inertia with dimensionless tidal deformability parameter $\bar{\lambda}$ as defined in Sec. 2.2 for $n p, n p \Lambda, n p \Lambda K^{-}$and $\mathrm{HQ}$ EoS. Like Fig. 6] we find again a universal relation in $\bar{I}-\bar{\lambda}$ for three 


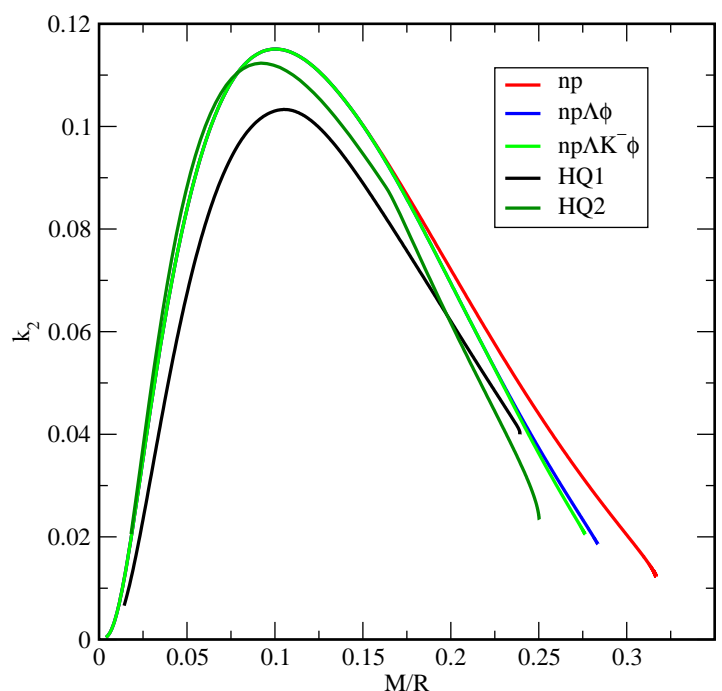

Fig. 9. Love number is plotted with compactness of neutron star.

hadronic EoSs. However, the data of HQ EoSs are deviating from the universal relation. It is now evident that Fig. 6 and Fig. 10 together produce I-Love-Q relations which are not sensitive to the compositions and EoS of neutron star matter without a first order phase transition. In those cases, neutron star matter below the saturation density is well constrained by nuclear physics experiments in laboratories. All EoSs in this density regime should behave in the same fashion and lead to universal relations. However, this is not true at higher densities where many new degrees of freedom appear in the form of hyperons, antikaon condensate and quarks. We find significant deviations in EoSs in that density regime. It is then a puzzle to understand what drives the universality when the neutron star compactness increases. This has been attributed to the isodensity contours in neutron stars which are approximately elliptically self similar [13,41]. It is worth mentioning here this kind of universal relations among I and Q were also found in rotating protoneutron stars [41,42].

\section{Conclusions}

We study properties of rotating neutron stars using LOREN as well as the Hartle-Thorne prescription of slowly rotating neutron stars. We exploit different EoSs involving hyperons, Bose-Einstein condensate of antikaons and quarks. Next we investigate the behaviour of moment of inertia, quadrupole moment and their dimensionless counter parts with gravitational mass and compactness of neutron stars. Furthermore, we study the Love number for different EoSs. We find that all those quantities are dependent on EoSs. It is also noted that the tidal Love number approaches a smaller value for large compactness. Similarly, the quadrupole moment of a rotating neutron star moves

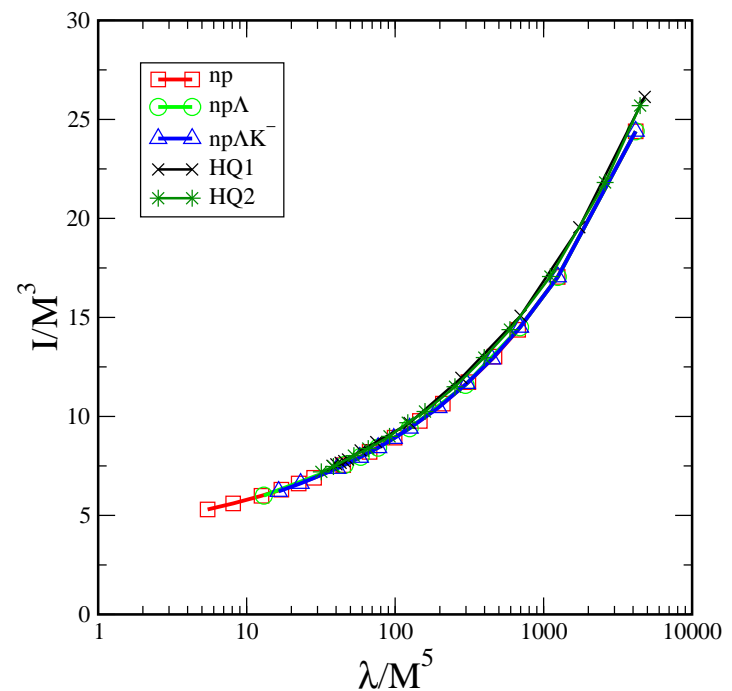

Fig. 10. Dimensionless moment of inertia is shown as a function of dimensionless deformability parameter $(\bar{\lambda})$

closer towards the Kerr value of a black hole for maximum mass neutron stars.

We further investigate the relations among dimensionless moment of inertia, quadrupole moment and tidal deformability parameter. The universal I-Q and I-Love number relations are observed in our calculation for EoS including hyperons and antikaon condensate as predicted by Yagi and Yunes [10. However, we have shown for the first time that the universality in I-Q relation is violated for HQ EoSs undergoing a first order phase transition.

\section{Acknowledgements}

DB has fond memories of his interactions with Walter Greiner.

\section{References}

1. J. Antoniadis et al., Science 340, (2013) 448.

2. M. Burgay et al., Nature 426, (2003) 531.

3. J. M. Lattimer and B. F. Schutz, ApJ 629, (2005) 979.

4. http://www.gw.iucaa.in/ligo-india/

5. P. Abbott et al., PRL 119, (2017) 161101.

6. D. Radice, S. Bernuzzi, W. D. Pozzo, L. F. Roberts and C. D. Ott, ApJ 842, (2017) L10.

7. E. E. Flanagan and T. Hinderer, Phys. Rev. D 77, (2008) 021502 .

8. T. Hinderer, ApJ 677, (2008) 1216.

9. T. Hinderer, B. D. Lackey, R. N. Lang and J.S. Read, Phys. Rev. D 81, (2010) 1230161.

10. K. Yagi and N. Yunes, Science 341, (2013) 365.

11. D. D. Doneva, S. S. Yazadjiev, N. Stergioulas, and K. D. Kokkotas, Astrophys. J. 781, L6 (2014).

12. B. Haskell, R. Ciolfi, F. Pannarale, and L. Rezzolla, Mon. Not. R. Astron. Soc. Lett. 438, L71 (2014). 
13. K. Yagi, N. Yunes, Phys. Rep. 681 (2017) 1-72

14. N. K. Glendenning, Phys. Lett. B 114, (1982) 392.

15. J. Schaffner and I. N. Mishustin, Phys. Rev. C 53, (1996) 1416.

16. S. Weissenborn, D. Chatterjee and J. Schaffner-Bielich, Nucl. Phys. A 881, (2012) 62.

17. D. Chatterjee and I. Vidaña, Eur. Phys. J. A 52, (2016) 29.

18. D. B. Kaplan and A. E. Nelson, Phys. Lett. B 175, (1986) 57.

19. R. Knorren, M. Prakash and P. J. Ellis, Phys. Rev. C 52, (1995) 3470.

20. E. Farhi and R. L. Jaffe, Phys. Rev. D 30, (1984) 2329.

21. S. Banik, M. Hempel and D. Bandyopadhyay, ApJS 214, (2014) 22.

22. P. Char and S. Banik, Phys. Rev. C 90, (2014) 015801.

23. S. Typel et al., Phys. Rev. C 81, (2010) 015803.

24. N. K. Glendenning and J. Schaffner-Bielich, Phys. Rev. D 46, (1999) 025803.

25. S. Banik and D. Bandyopadhyay, Phys. Rev. C 64, (2001) 055805 .

26. R. D. Mellinger Jr, F. Weber, W. Spinella, G. A. Contrera and M. G. Orsaria, Universe, 3, (2017) 1.

27. We use the hadron-quark (HQ) EoS table of Fridolin Weber (private communication).

28. I. N. Mishustin, R. Mallick, R. Nandi and L. Satarov, Phys. Rev. C, 91, (2015) 055806.

29. M. Orsaria, H. Rodrigues, F. Weber and G. A. Contrera, Phys. Rev. D, 87, (2013) 023001.

30. S. Bonazzola, E. Gourgoulhon, M. Salgado and J.-A. Marck, Astron. Astrophys. 278, 421 (1993).

31. M. Salgado, S. Bonazzola, E. Gourgoulhon, and P. Haensel, Astron. Astrophys. 291, 155 (1994).

32. E. Gourgoulhon, P. Grandclément, J.-A. Marck, J. Novak and K. Taniguchi, "LORENE spectral methods differential equation solver", Astrophysics Source Code Library ascl:1608.018, (2016).

33. http://www.lorene.obspm.fr/

34. G. Pappas and T.A. Apostolatos, Phys. Rev. Lett. 108, 231104 (2012)

35. J. L. Friedman and N. Stergioulas, Rotating Relativistic Stars, Cambridge University Press, 2013

36. K. Yagi and N. Yunes, Phys. Rev. D 88, (2013) 023009.

37. F. Cipolletta, C. Cherubini, S. Filippi, J.A. Rueda and R. Ruffini, Phys. Rev. D 92, (2015) 023007.

38. M. Urbanec, J. C. Miller and Z. Stuchlík, MNRAS 443, (2013) 1903.

39. C. Breu and L. Rezzolla, MNRAS 459, (2016) 646 .

40. S. S. Lenka, P. Char and S. Banik, Int. J. Mod. Phys. 26, (2017) 1750127

41. G. Martinon, A. Maselli, L. Gualtieri and V. Ferrari, Phys. Rev. D 90, (2014) 064026.

42. M. Marques, M. Oertel, M. Hempel and J. Novak, Phys. Rev. C 96, (2017) 045806. 